\title{
Inhibition of MUS81 improves the chemical sensitivity of olaparib by regulating MCM2 in epithelial ovarian cancer
}

\author{
AILING ZHONG ${ }^{1,2^{*}}$, HONGQIN ZHANG $^{1,2^{*}}$, SUHONG XIE $^{1}$, MINJIE DENG $^{1}$, HUI ZHENG $^{1}$, \\ YANCHUN WANG ${ }^{1}$, MIAOMIAO CHEN ${ }^{1,2}$, RENQUAN LU ${ }^{1,2}$ and LIN GUO ${ }^{1,2}$ \\ ${ }^{1}$ Department of Clinical Laboratory, Fudan University, Shanghai Cancer Center; ${ }^{2}$ Department of Oncology, \\ Shanghai Medical College, Fudan University, Shanghai, P.R. China
}

Received October 10, 2017; Accepted January 17, 2018

DOI: $10.3892 /$ or.2018.6229

\begin{abstract}
Dysfunction of the DNA repair pathway contributes to tumorigenesis and drug resistance. Methyl methanesulfonate and ultraviolet sensitive gene clone 81 (MUS81), a key endonuclease in DNA repair, is generally considered a tumor suppressor; however, recent studies have revealed its tumorpromoting effect in epithelial ovarian cancer (EOC) and have shown that its overexpression is associated with cisplatin sensitization. However, the exact functional role of MUS81 and its regulation in relation to chemotherapy sensitivity remains unknown. Our previous study using protein interaction chip revealed that minichromosome maintenance complex component 2 (MCM2) is closely correlated with MUS81. This study aimed to investigate the biological effects and mechanisms of MUS81 on cellular responses to chemotherapeutic drugs. To accomplish this, we downregulated MUS81 and MCM2 in A2780 and SKOV3 ovarian cancer cells using lentivirus-mediated RNAi. Using a qPCR-based HR assay kit to detect HR efficiency. The sensitivity of MUS81 to olaparib was investigated by cell proliferation, colony formation assays and flow cytometry. The results showed that MUS81 modulates MCM2 levels as well as homologous recombination (HR) activity. Moreover, downregulation of MUS81 increased the sensitivity of EOC cells to olaparib by inducing $S$ phase arrest and promoting apoptosis through activation of MCM2. MUS81 may be a potential novel therapeutic target for EOC.
\end{abstract}

Correspondence to: Professor Lin Guo or Professor Renquan Lu, Department of Clinical Laboratory, Fudan University, Shanghai Cancer Center, 270 Dongan Road, Xuhui, Shanghai, P.R. China

E-mail: guolin500@hotmail.com

E-mail: lurenquan@126.com

*Contributed equally

Key words: MUS81, MCM2, homologous recombination, PARP inhibitors, epithelial ovarian cancer

\section{Introduction}

Ovarian cancer is one of the most common malignant tumors of the gynecologic system. In particular, epithelial ovarian cancer (EOC) is the most common type of ovarian cancer and is a serious gynecological disease due to its asymptomatic nature, accounting for $50-70 \%$ of ovarian cancers (1). Platinum and paclitaxel are the most frequently used chemotherapeutic agents for treatment of EOC. However, frequent recurrence and drug resistance often affect therapeutic outcomes.

Methyl methanesulfonate and ultraviolet sensitive gene clone 81 (MUS81), which is critical for the repair of DNA double-strand breaks (DSBs), is a highly conserved gene across species and encodes a structure-specific DNA endonuclease $(2,3)$. DNA DSBs resolve Holliday junctions (HJs) by constituting a heterodimer with Eme1/Mms4 and maintaining genetic stability (4). HJs are co-regulated by the BTR (BLM-topoisomerase IIIa-RMI1-RMI2) complex, the SLX-MUS (SLX1-SLX4-MUS81-EME1) complex and GEN1 (5). Changes in HJs affect both the activity and DNA stability of homologous recombination (HR) in the repair of DSBs. Our previous studies demonstrated that downregulation of MUS81 inhibits ovarian tumor growth and enhances platinum drug sensitivity both in vivo and in vitro (6). Moreover, MUS81-targeting represents a novel approach for promoting sensitivity to chemotherapy and has recently attracted significant research attention. Studies have reported that embryonic stem cells and mice that are deficient in the MUS81 gene are significantly more sensitive to mitomycin C (MMC). The survival of mice with MUS81 ${ }^{+/-}$and MUS81 ${ }^{-/}$genotypes was significantly lower than that of wild-type mice treated with the same dose of MMC (7). Disruption of the MUS81 gene increases sensitivity of MMC and cisplatin, returning to normal after MUS81 is re-expressed. These results indicate that MUS81 plays an essential role in the chemosensitivity of human malignancies (8). Although previous studies have found that MUS81 is associated with chemotherapeutic drug sensitivity in ovarian cancer and potentially inhibits HR activity via MUS81 depletion, the role of MUS81 in regulating chemosensitivity in ovarian cancer remains unclear.

To overcome the challenge of resistance to chemotherapy agents, poly(ADP-ribose) polymerase PARP inhibitors have been applied for the treatment of BRCA-mutated ovarian 
cancer and have resulted in satisfactory treatment effects $(9,10)$. Recent studies found that, olaparib, a potent oral PARP inhibitor, can be used as a targeted therapeutic drug for BRCA-mutated ovarian cancer treatment $(11,12)$. The antitumor effect of olaparib was demonstrated in ovarian cancer and was associated with HR deficiency (13). However, the role of olaparib in the treatment of BRCA wild-type ovarian cancer, potentially via the modulation of pathways to induce HR deficiency, has not yet been fully elucidated. In this study, the role of MUS81 on the chemosensitivity of olaparib in EOC, its underlying molecular mechanisms and its association with HR were investigated.

\section{Materials and methods}

Cell lines. Human ovarian cancer A2780 and SKOV3 cells were purchased from the Chinese Academy of Sciences Committee (Shanghai, China). All ovarian cells were cultured in RPMI-1640 medium supplemented with $10 \%$ fetal bovine serum (FBS) and 1\% penicillin-streptomycin. Cells were incubated at $37^{\circ} \mathrm{C}$ in a humidified atmosphere with $5 \% \mathrm{CO}_{2}$.

Immunofluorescence. For immunofluorescence studies, $5 \times 10^{5}$ cells were seeded in 24-well plates on glass coverslips. After the cells adhered, they were washed with PBS and fixed with $4 \%$ formaldehyde at room temperature. The fixed cell slides were washed with PBS, and then methanol and acetone were added at a 1:1 ratio, followed by blocking with $1 \%$ BSA. The cells were then incubated with antibodies against MUS81 (Santa Cruz Biotechnology, Inc., Dallas, TX, USA) and MCM2 [Cell Signaling Technology, (CST) Inc., Beverly, MA, USA)] overnight at $4^{\circ} \mathrm{C}$. After washing, the cells were labeled with Alexa Fluor 488-conjugated secondary antibody (Invitrogen, Carlsbad, CA, USA) and examined under a fluorescence microscope (SP5; Leica Microsystems, Wetzlar, Germany).

Co-immunoprecipitation. Cultured cells were carefully washed twice with $4^{\circ} \mathrm{C} \mathrm{PBS}$, and IP lysis buffer (Beyotime Biotechnology, Shanghai, China) was added. To extract protein, cells were scraped off, and 50\% protein A/G agarose (Santa Cruz Biotechnology, Inc.) was added to the sample solution at a ratio of 1:10. The cells were placed on a horizontal shaker for $1 \mathrm{~h}$ at $4^{\circ} \mathrm{C}$. Then, the protein A/G-agarose beads were discarded and the appropriate amount of primary MUS81 antibody was added according to the protein content. The antigen-antibody complex was slowly shaken in a rotating shaker at $4^{\circ} \mathrm{C}$ overnight. The antigen-antibody complexes were precipitated with protein $\mathrm{A} / \mathrm{G}$-agarose beads, and the supernatant was collected for western blotting.

Cells treated with $U V$ and CPT. Cells were seeded in 6-well plates at a density of $4 \times 10^{5}$ cells/well. CPT (20/40/80 nmol/l) was added to A2780 and SKOV3 cells, and a well without CPT was taken as a control, and the cells were cultured for $24 \mathrm{~h}$. Cells were collected and lysed for total protein extraction using IP lysis buffer (Beyotime Biotechnology). The expression of MUS81 and MCM2 were verified by western blotting, and $\mathrm{pH} 2 \mathrm{AX}$ was used as a sign of the repair of double-strand breaks. The cells were irradiated with different degrees of ultraviolet radiation using a UV cross-linking instrument. The irradiation degree was calculated as number $\mathrm{x}$ area $\mathrm{x} 1 \times 10^{6}$. Protein was extracted using the same method and verified with western blotting.

Establishment of cell lines with downregulation of MUS81 and MCM2. A MUS81 lentivirus expressing a specific RNAi interference sequence was first designed. The nucleotide sequences were cloned into the AgeI and EcoRI sites of the pLKO.1puro vector (Addgene, Cambridge, MA, USA) to generate the pLKO.1-puro-MUS81 (shMUS81-1 and shMUS81-2) and pLKO.1-puro-control (shCtrl) (Table I) recombinant vectors. Lentiviruses were packaged by transfecting $293 \mathrm{~T}$ cells with the above lentivirus recombinant vectors, the packing plasmid psPAX2, and the envelop vector pMD2.G (Addgene) using Lipofectamine 2000 transfection reagent (Invitrogen). Culture medium containing lentivirus particles was collected after $48 \mathrm{~h}$. Ovarian cancer cells were transfected with $1 \times 10^{6} \mathrm{IFU} / \mathrm{ml}$ lentivirus in $8 \mathrm{mg} / \mathrm{ml}$ Polybrene (Sigma-Aldrich, St. Louis, MO, USA) for $24 \mathrm{~h}$. Stably transduced cells were selected using $1 \mathrm{mg} / \mathrm{ml}$ puromycin (Sigma-Aldrich) for 7 days, and the RNAi knockdown efficiency was detected by western blotting. A2780 cells with MCM2 downregulation were established via the same method.

Western blotting. Total protein was collected as described above. Cell lysates were resolved by SDS-PAGE, and proteins were electro-transferred to polyvinylidene fluoride (PVDF) membranes (Millipore, USA). The PVDF membranes were blotted with $10 \%$ non-fat milk (Solarbio, Beijing, China). The primary antibodies included MUS81 (1:200 dilution; Santa Cruz Biotechnology, Inc.), $\beta$-actin (1:3,000 dilution; Abcam, Cambridge, MA, USA), pH2AX (1:400 dilution; CST), cyclin B (1:3,000 dilution; Abcam), POLE (1:200 dilution; Santa Cruz Biotechnology, Inc.), NBS1 (1:3,000 dilution; Abcam) and MCM2 (1:1,000 dilution; CST).

Quantitative RT-PCR. Preparation of MUS81 and MCM2 gene downregulated cell lines. RNA was isolated from cells using TRIzol $^{\circledR}$ reagent (Thermo Fisher Scientific, Shanghai, China) and reverse transcribed using a PrimeScript ${ }^{\circledR}$ RT reagent kit (Takara, Shiga, Japan) according to the manufacturer's protocol. RT-PCR was performed in a $10 \mu 1$ reaction solution containing $10 \mathrm{ng}$ cDNA, $0.1 \mathrm{mmol} / \mathrm{l}$ primer (Table I), and $5 \mathrm{ml}$ 2X SYBR Premix Ex Taq (Takara). PCR amplification was performed at $95^{\circ} \mathrm{C}$ for $5 \mathrm{~min}$, followed by 40 cycles at $95^{\circ} \mathrm{C}$ for $15 \mathrm{sec}$, and $65^{\circ} \mathrm{C}$ for $40 \mathrm{sec}$, using a Mastercycler ${ }^{\circledR}$ ep realplex (Quantstudio dx, Thermo Fisher Scientific). The abundance of MUS81 and MCM2 transcripts was expressed relative to $\beta$-actin as a control.

Measurement of HR efficiency. HR efficiency was detected using a qPCR-based HR assay kit (Norgen BiotekCorp., Thorold, ON, Canada). The dl-1 and dl-2 plasmids were co-transfected into shCtrl, shMUS81-1 and shMUS81-2 cells, and the total cellular DNA was isolated using a Blood \& Cell Culture DNA Mini kit (Qiagen, Hilden, Germany) after $48 \mathrm{~h}$. qPCR was performed using a set of universal primers and the assay primer. The difference in the number of cycles to reach the amplification curve inflection point generated using HR-specific primers and the number of cycles to reach 
Table I. Sequences of primers and targets.

Primers/targets

Sequences

MUS81 qRT-PCR forward

MUS81 qRT-PCR reverse

$\beta$-actin qRT-PCR forward

$\beta$-actin qRT-PCR reverse

MCM2 qRT-PCR forward

MCM2 qRT-PCR reverse

shMUS81-1

shMUS81-2

shCtrl/SRC (scrambled sequence)

MCM2-1

MCM2-3

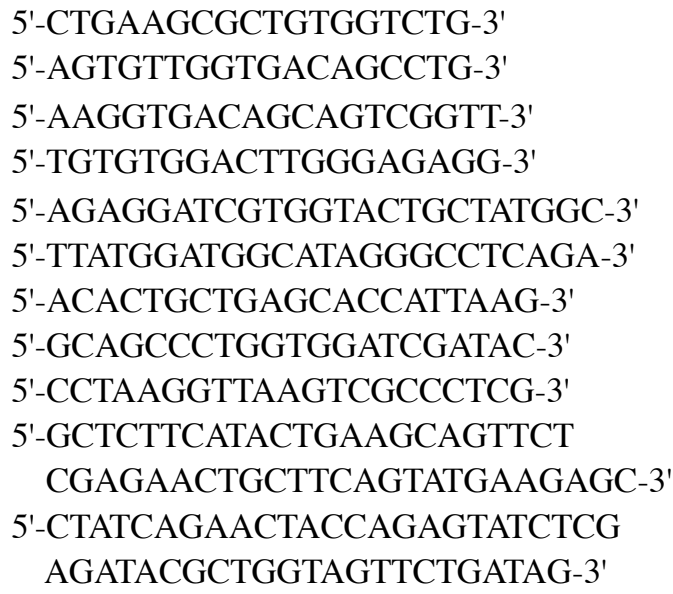

5'-CTGAAGCGCTGTGGTCTG-3'

5'-AGTGTTGGTGACAGCCTG-3'

5'-AAGGTGACAGCAGTCGGTT-3'

5'-TGTGTGGACTTGGGAGAGG-3'

5'-AGAGGATCGTGGTACTGCTATGGC-3'

5'-TTATGGATGGCATAGGGCCTCAGA-3'

5'-ACACTGCTGAGCACCATTAAG-3'

5'-GCAGCCCTGGTGGATCGATAC-3'

5'-CCTAAGGTTAAGTCGCCCTCG-3'

5'-GCTCTTCATACTGAAGCAGTTCT

CGAGAACTGCTTCAGTATGAAGAGC-3'

5'-CTATCAGAACTACCAGAGTATCTCG

AGATACGCTGGTAGTTCTGATAG-3'

Methyl methanesulfonate and ultraviolet sensitive gene clone 81; MCM2, minichromosome maintenance complex component 2.

the universal primer amplification curve inflection point was converted to the difference in DNA content based on a standard DNA content curve. This value was set to 1 for the control cell populations, and the amount of recombinant HR DNA produced in the experimental groups was normalized to this value.

Olaparib sensitivity assay. Single-cell suspensions were prepared and seeded in 96-well plates ( $1 \times 10^{4}$ cells/well). After treatment with serial dilutions of olaparib (MCE, Shanghai, China) ranging from 2 to $10 \mu \mathrm{mol} / 1$ for $48 \mathrm{~h}$, cell viability was assessed using a CCK-8 kit (Dojindo, Kumamoto, Japan). Each well was read at a wavelength of $450 \mathrm{~nm}$ (Synergy H4; Bio-Tek). Cell viability was calculated as follows: Viability of cells $(\%)=($ drug group-blank) OD450/(no drug group-blank) OD450 x $100 \%$.

Colony formation assay. For the colony formation assay, 1,000 cells/well were seeded into 6 -well plates and incubated for 10-14 days until visible clones appeared. The control group was cultured in RPMI-1640 medium supplemented with $10 \%$ FBS and $1 \%$ penicillin-streptomycin, and the experimental group was treated with $5 \mu \mathrm{mol} / 1$ olaparib. Colonies were stained with $0.5 \%$ crystal violet. The number of colonies was determined using a microscope.

Cell cycle and apoptosis assay. Cells were seeded onto 6-well plates at a density of $4 \times 10^{5}$ cells/well, and then treated with olaparib $(5 \mu \mathrm{mol} / \mathrm{ml})$ for $48 \mathrm{~h}$. To examine apoptosis, $1 \times 10^{5}$ cells were collected and washed twice with $4^{\circ} \mathrm{C}$ PBS. Then, the cells were resuspended in $1 \mathrm{X}$ Annexin $\mathrm{V}$ binding buffer and stained with propidium iodide (PI) and Annexin V according to the instructions of the Annexin V-fluorescence apoptosis detection kit I (BD Biosciences; Pharmingen, San Diego, CA, USA). For cell cycle analysis, transduced cells were harvested, fixed in $70 \%$ alcohol overnight at $4^{\circ} \mathrm{C}$, and then incubated with $500 \mathrm{ml}$ of PI (BD Pharmingen ${ }^{\mathrm{TM}}$ ) for $15 \mathrm{~min}$ in the dark. Finally, apoptosis and the cell cycle were analyzed by flow cytometry (BD, Caliburn), and the assays were repeated three times.

Statistical analysis. Data are presented as the mean \pm SD of three independent experiments, and SPSS 17.0 software was used for statistical analyses. The differences between groups were examined by analysis of variance (ANOVA) and Student's t-tests. $\mathrm{P}<0.05$ was considered to indicate statistical significance.

\section{Results}

MUS81 interacts with MCM2 to participate in the DNA damage repair process. MUS81 induces an important endonuclease effect on the repair of damaged replication forks of HJ. MCM2, a pivotal component of MCM2-7 helicase, induces helicase activity at the replication fork and regulates the formation of DNA double strands with downstream molecules. In this study, we found that both MUS81 and MCM2 were localized in the nucleus, as shown by immunofluorescence assays (Fig. 1A). Our previous data generated using protein interaction chips revealed five proteins that bind to MUS81, of which MCM2 had the highest correlation. In A2780 cells, MUS81 co-existed with MCM2 and POLE in co-immunoprecipitation experiments, and therefore, we speculated that the interaction between MUS81 and MCM2 is regulated by POLE (Fig. 1B). To study the roles of MUS81 and MCM2 in DSB repair in vitro, a DNA DSB model was established via physical treatment with UV irradiation, and chemical treatment with camptothecin (CPT). The results indicated that DNA DSBs occurred after $24 \mathrm{~h}$ of UV irradiation, at which time MUS81 and MCM2 were upregulated with increasing degrees of UV irradiation-induced damage (Fig. 2A). After $1 \mathrm{~h}$ of irradiation, nuclear MUS81 and MCM2 were involved in the process of repairing gene damage, resulting in no rupture of the DNA double strand. CPT treatment can lead to DNA DBSs in cells and induce the formation of replication forks, which can be 

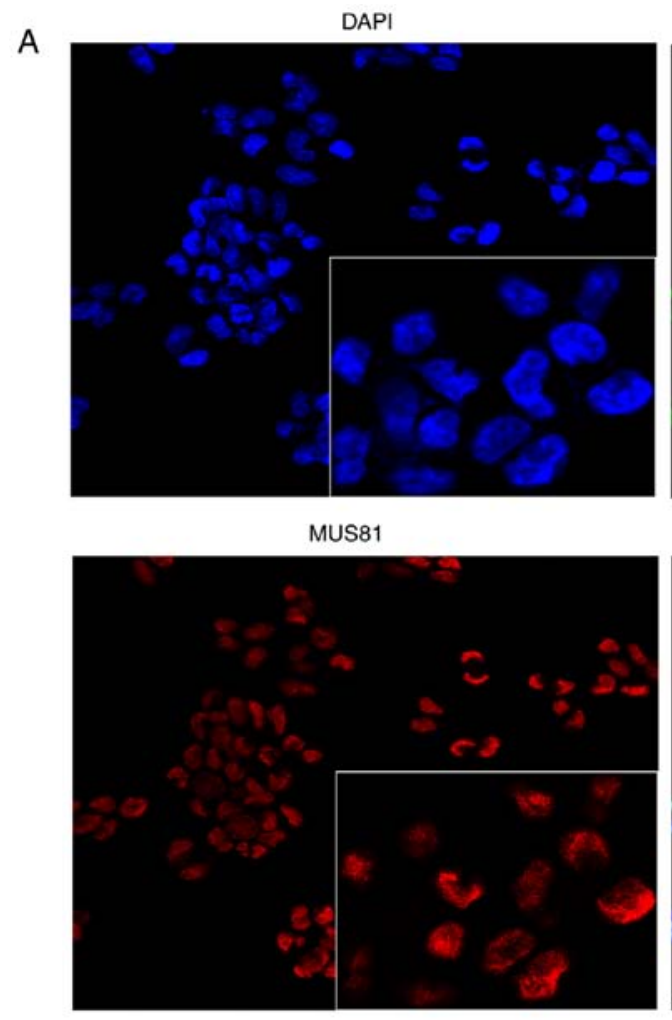

B

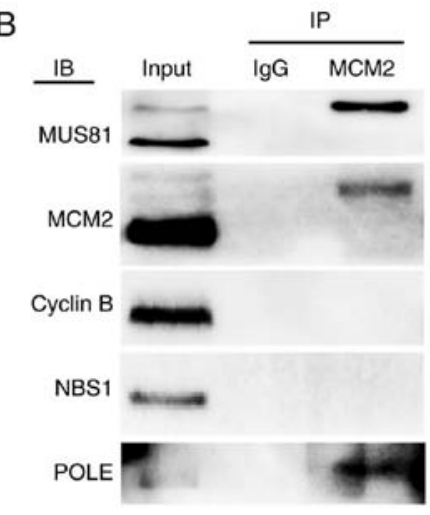

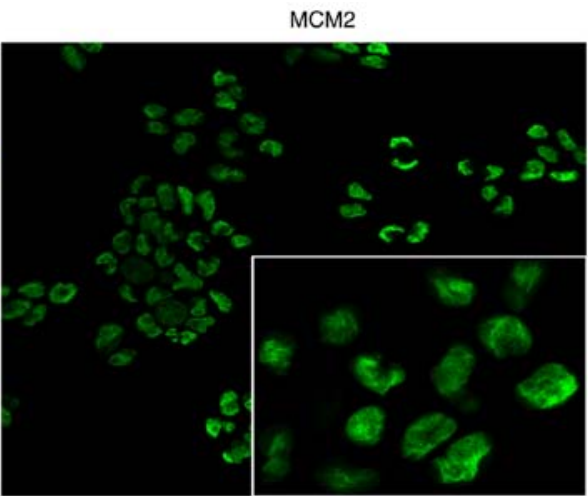
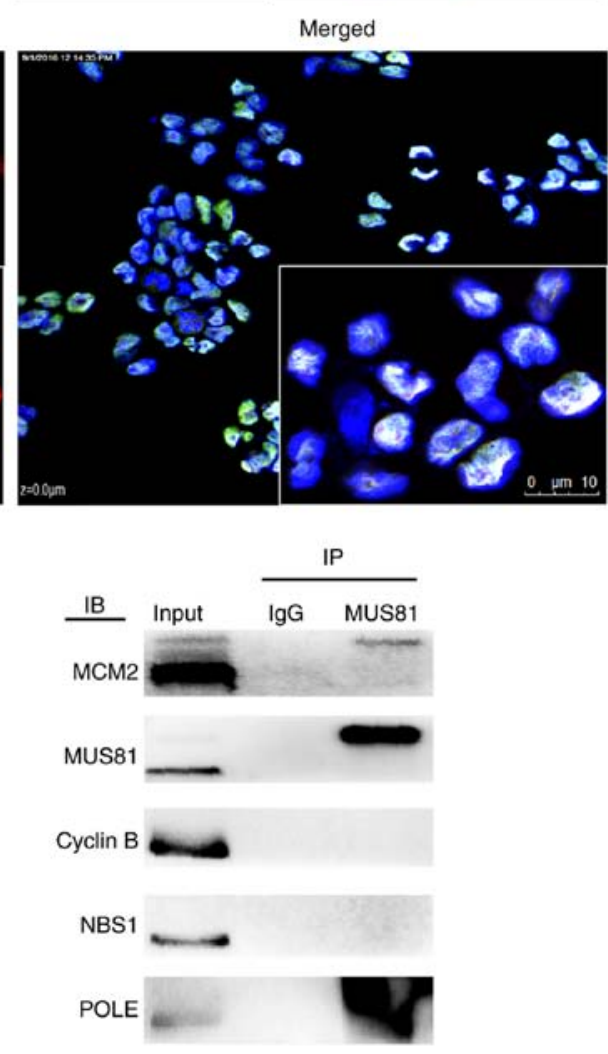

Figure 1. MUS81 and MCM2 co-expression and interaction in the nucleus. (A) Confocal microscopy revealed co-localization of MUS81 and MCM2 protein in A2780 cells. (B) Cell lysates were denatured and then immunoprecipitated with MUS81 or MCM2 antibodies. Both forward and reverse immunoprecipitation showed that MUS81 bound to MCM2.

assessed by measuring $\mathrm{P}-\mathrm{H} 2 \mathrm{AX}$ as an indicator of represent damage repair. MCM2 and MUS81 expression in A2780 and SKOV3 cells dose-dependently increased with CPT treatment after $24 \mathrm{~h}$ (Fig. 2B), suggesting that both MUS81 and MCM2 are involved in the formation of replication forks and HR.

HR activity decreases after MUS81 downregulation, and there is negative feedback regulation between MUS81 and MCM2. A2780 and SKOV3 cells with downregulation of MUS81 expression were constructed using a lentivirus-mediated method, and the regulatory relationship between MUS81 and MCM2 at the protein level in ovarian cancer was further investigated in vitro. Increased expression of MCM2 was found in shMUS81-1 and shMUS81-2 ovarian cells compared with shCtrl cells according to western blotting, and MUS81 played a negative role in the regulation of MCM2. Consistent results were observed at the RNA level by RT-PCR (Fig. 3A). To further define the regulatory relationship between MUS81 and MCM2, we constructed MCM2-downregulated A2780 cell lines and found that MUS81 levels decreased following the downregulation of MCM2 (Fig. 3B). In accordance with these results, we hypothesized that $\mathrm{MCM} 2$, as a molecule upstream of MUS81, is involved in regulating MUS81 expression levels and that MUS81 expression exerts negative feedback regulation on MCM2. STRING database results revealed that POLE may regulate the interaction between MUS81 and MCM2 (Fig. 3C). As the main factor of HJ decomposition and involved in HR, the association of MUS81 with HR activity was further investigated. A significant reduction in the HR efficiency of shMUS81 cells compared with shCtrl cells was observed via qPCR using an HR assay kit $(\mathrm{P}<0.05)$ (Fig. 3D). These results suggested that MUS81 and MCM2 are involved in HR of DSBs. The suspension of HJ formation and dissociation of the replication fork may be inhibited by the activation of 
A

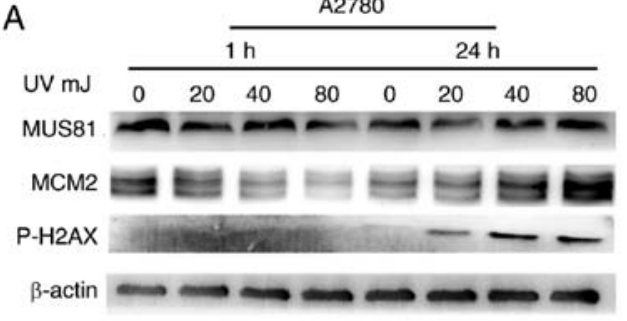

B

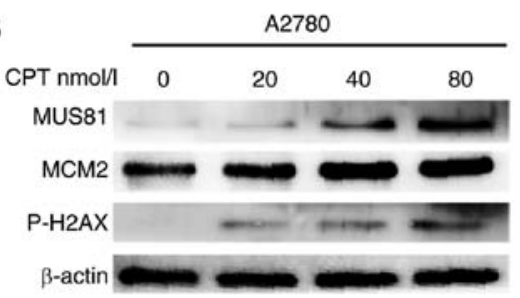

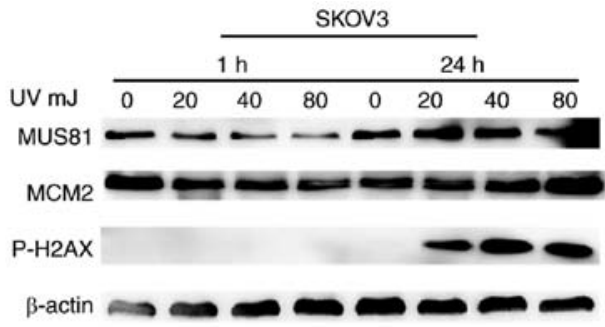

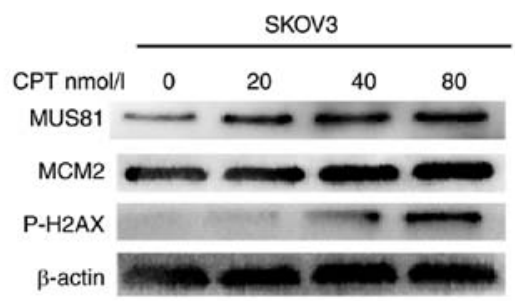

Figure 2. DNA double strand break damage and repair model. (A and B) Different degrees of DNA damage caused by UV irradiation and various concentrations of CPT, and western blot assays of MUS81 and MCM2 expression levels in A2780 and SKOV3 cells. P-H2AX was used as an indicator of DSBs.

A

A
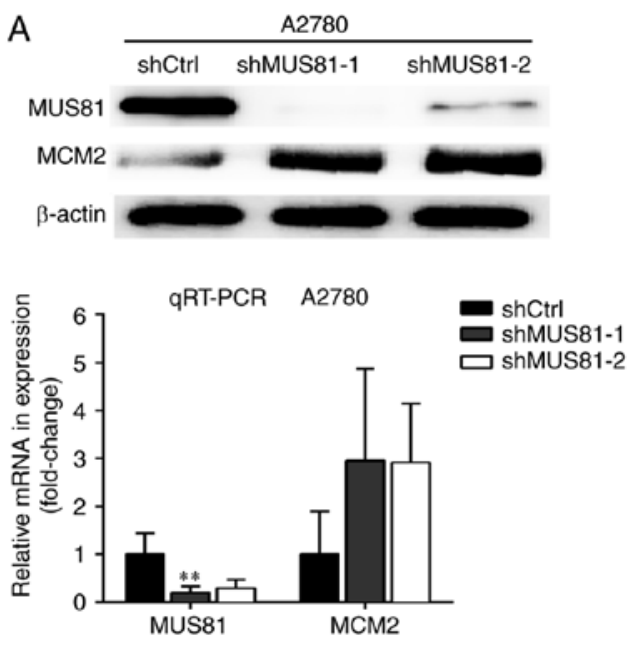

B

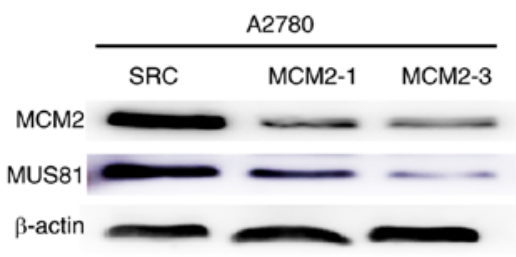

C

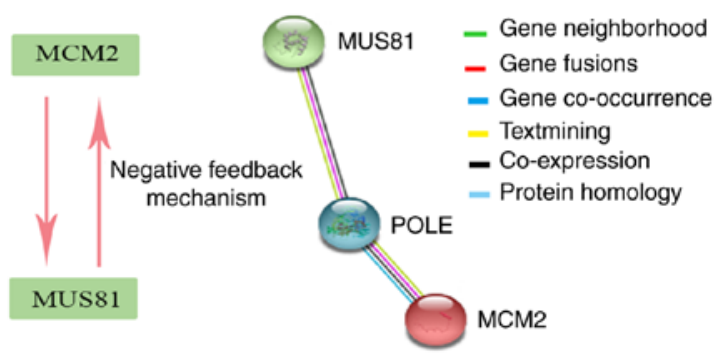

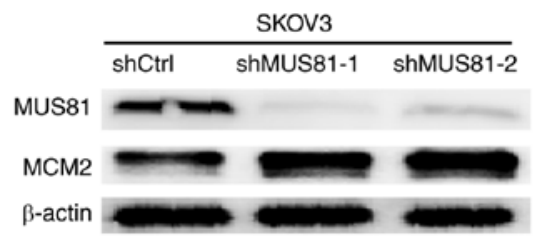
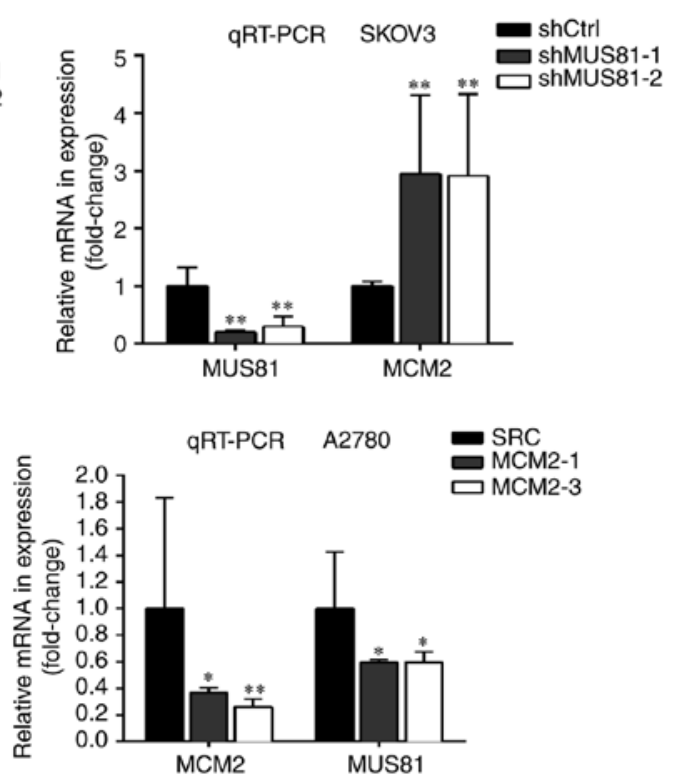

D Homologous recombination assay

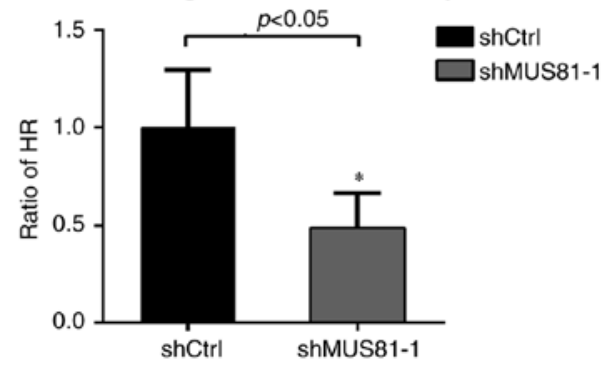

Figure 3. MUS81 regulates MCM2 via negative feedback and reduces HR activity. (A) Lentivirus-mediated RNAi constructs were used to generate the MUS81-downregulated A2780 and SKOV3 cell lines, and increased expression of MCM2 was revealed by western blotting and RT-PCR. (B) The MCM2 downregulated A2780 cell line was constructed using the same method and analyzed by western blotting and RT-PCR. ("P<0.05, $\left.{ }^{* *} \mathrm{P}<0.01\right)$. (C) MUS81 and MCM2 exhibited a negative regulatory relationship. (D) Among A2780 ovarian cancer cells lines, the HR efficiency of shMUS81-1 cells was significantly lower than that of shCtrl cells. $(\mathrm{P}<0.05)$. 

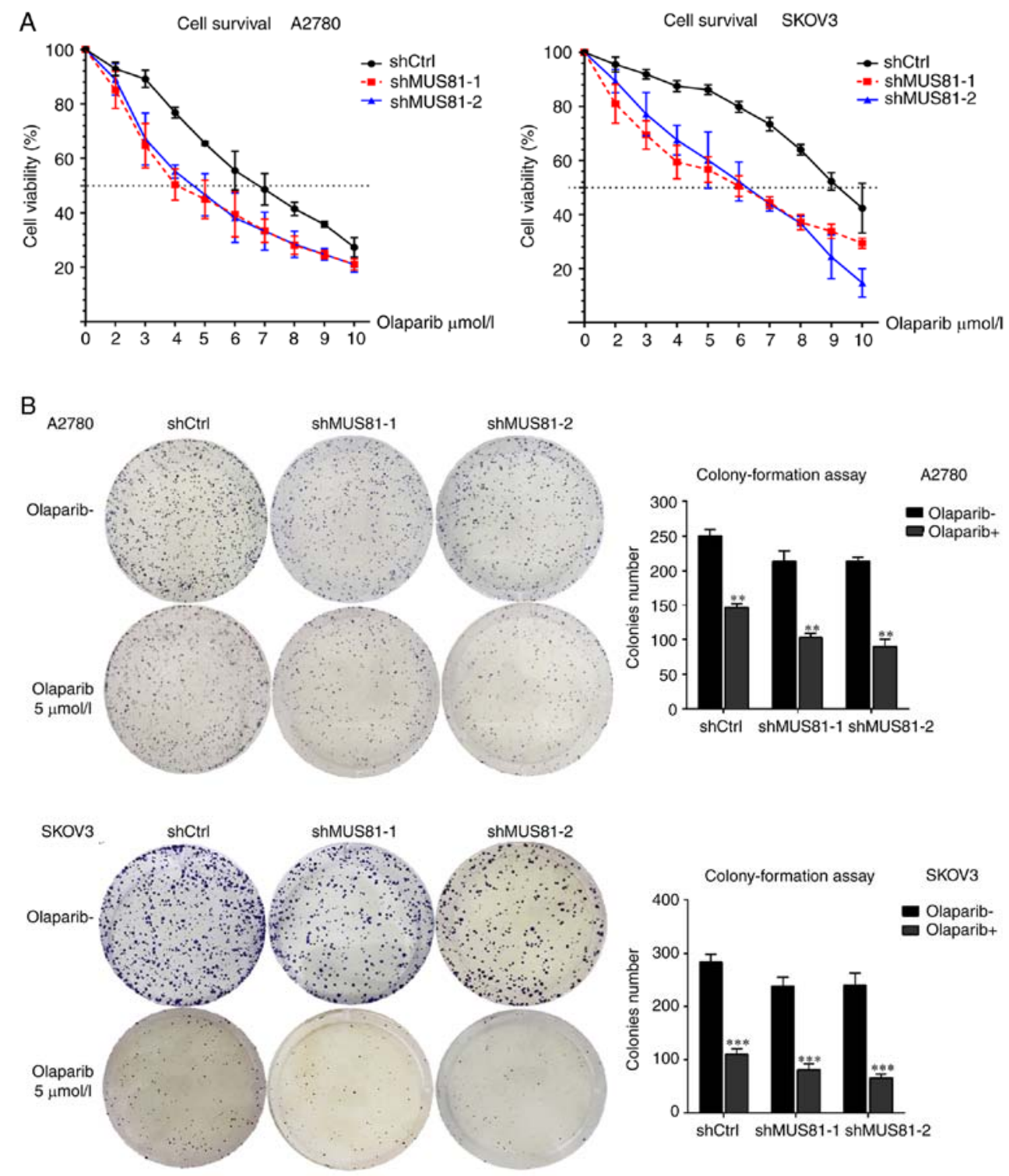

Figure 4. (A) Olaparib inhibits the growth of MUS81-downregulated cells. The $\mathrm{IC}_{50}$ values for olaparib in shMUS81-1 cells (A2780 IC ${ }_{50}, 4.56 \mu \mathrm{mol} / 1$; $\mathrm{SKOV} 3$ $\left.\mathrm{IC}_{50}, 5.63 \mu \mathrm{mol} / 1\right)$ and shMUS81-2 cells (A2780 IC $50,4.79 \mu \mathrm{mol} / 1$; SKOV3 $\left.\mathrm{IC}_{50}, 5.68 \mu \mathrm{mol} / 1\right)$ were significantly lower than those in shCtrl cells (A2780 $\mathrm{IC}_{50}, 6.74 \mu \mathrm{mol} / 1$; SKOV3 $\mathrm{IC}_{50}, 10.2 \mu \mathrm{mol} / 1$ ) as assessed by CCK-8 assays. The data are presented as the mean $\pm \mathrm{SD}$. (B) Downregulation of MUS81 increases drug sensitivity to olaparib. Olaparib treated SKOV3 and A2780 cells were assessed by colony formation assays. shMUS81-1 and shMUS81-2 cells were compared to shCtrl cells, and the data are presented as the mean \pm SD of three independent experiments. ${ }^{* *} \mathrm{P}<0.01,{ }^{* * * *} \mathrm{P}<0.001$.

MCM2 helicase function through downregulation of MUS81 and subsequent inhibition of HR activity.

Downregulation of MUS81 induces HR deficiency that promotes olaplarib-mediated inhibition of ovarian cancer cell growth. We found that olaparib acts on BRCA wild-type ovarian tumor cells with HR pathway defects and increases sensitivity to PARP inhibitors. No BRCA gene mutation was found in A2780 and SKOV3 cell lines in the COSMIC database. In MUS81-downregulated ovarian cancer cells, olaparib dose-dependently inhibited cell growth and significantly increased drug sensitivity in shMUS81-1 and shMUS81-2 cells compared to shCtrl cells (Fig. 4A). Furthermore, we performed colony formation assays and found significantly fewer stably transduced shMUS81-1/shMUS81-2 colonies than shCtrl cell colonies after treatment with olaparib $(\mathrm{P}<0.05)$ (Fig. 4B). These results indicate an impairment of the repair process in ovarian cancer cells resulting from HR deficiency mediated DSBs produced by the inhibition of MUS81 in BRCA wild-type cells.

Increased MCM2 expression resulting from MUS81 downregulation enhances drug sensitivity to olaparib. To investigate the mechanism of MUS81 activity on enhancing drug sensitivity to olaparib through the negative regulation of MCM2, flow cytometry was implied for cell cycle and apoptosis analysis. According to western blotting, $5 \mu \mathrm{mol} / 1$ olaparib inhibited MUS81 expression in shCtrl and shMUS81 ovarian cancer cells. The expression of MCM2 in shMUS81 cells significantly increased compared with shCtrl cells (Fig. 5A). Cell cycle analysis demonstrated that PARP inhibitor treatment of the stably transfected shMUS81 SKOV3 and A2780 cells induced significant $S$ phase arrest, and the percentage of G0/G1 phase cells significantly decreased (Fig. 5B). 

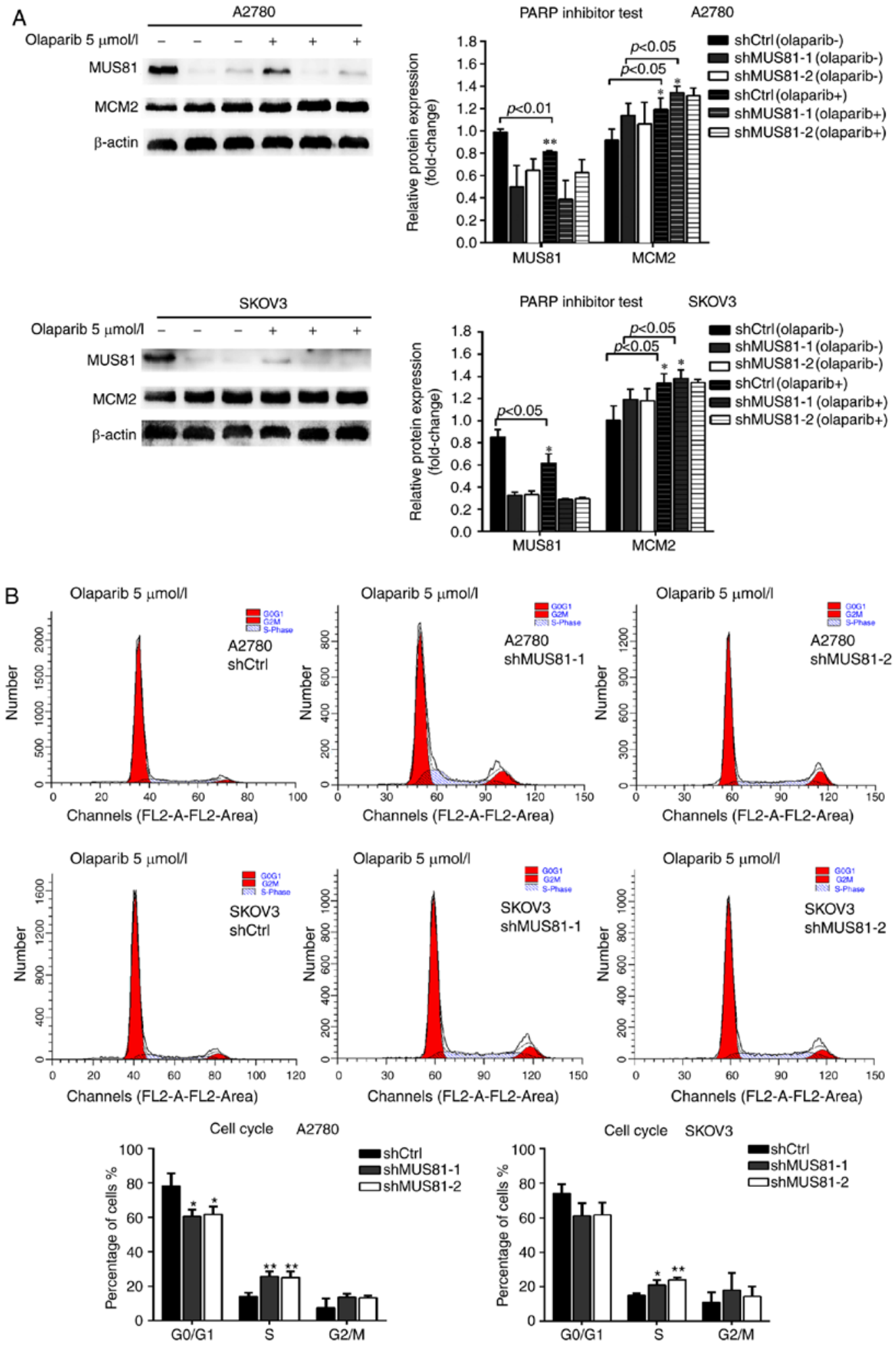

Figure 5. (A) The effect of olaparib on protein expression. Western blot assays of MUS81 and MCM2 expression levels in A2780 and SKOV3 cells after treatment with $5 \mu \mathrm{mol} / 1$ olaparib compared with a blank control. $\beta$-actin was used as the loading control. The bars (fold change) represent the relative expression of target protein to $\beta$-actin. (B) The effect of olaparib on cell cycle distribution. shCtrl and shMUS81 cells were exposed to $8 \mu \mathrm{mol} / 1 \mathrm{olaparib}$ for $48 \mathrm{~h}$. A2780 and SKOV3 cell cycle were analyzed by flow cytometry, and the data are presented as the mean \pm SD of three independent experiments.

Apoptosis assay also showed that olaparib treatment significantly increased apoptosis of shMUS81 A2780 and SKOV3 cells (Fig. 6). These results suggested that inhibition of MUS81 activates MCM2 expression and arrests cells in the
S phase. Consistent with previous studies, MCM2 is involved in promoting apoptosis. We confirmed that downregulation of MUS81 can enhance the sensitivity of BRCA wild-type ovarian cancer cells to olaparib. 

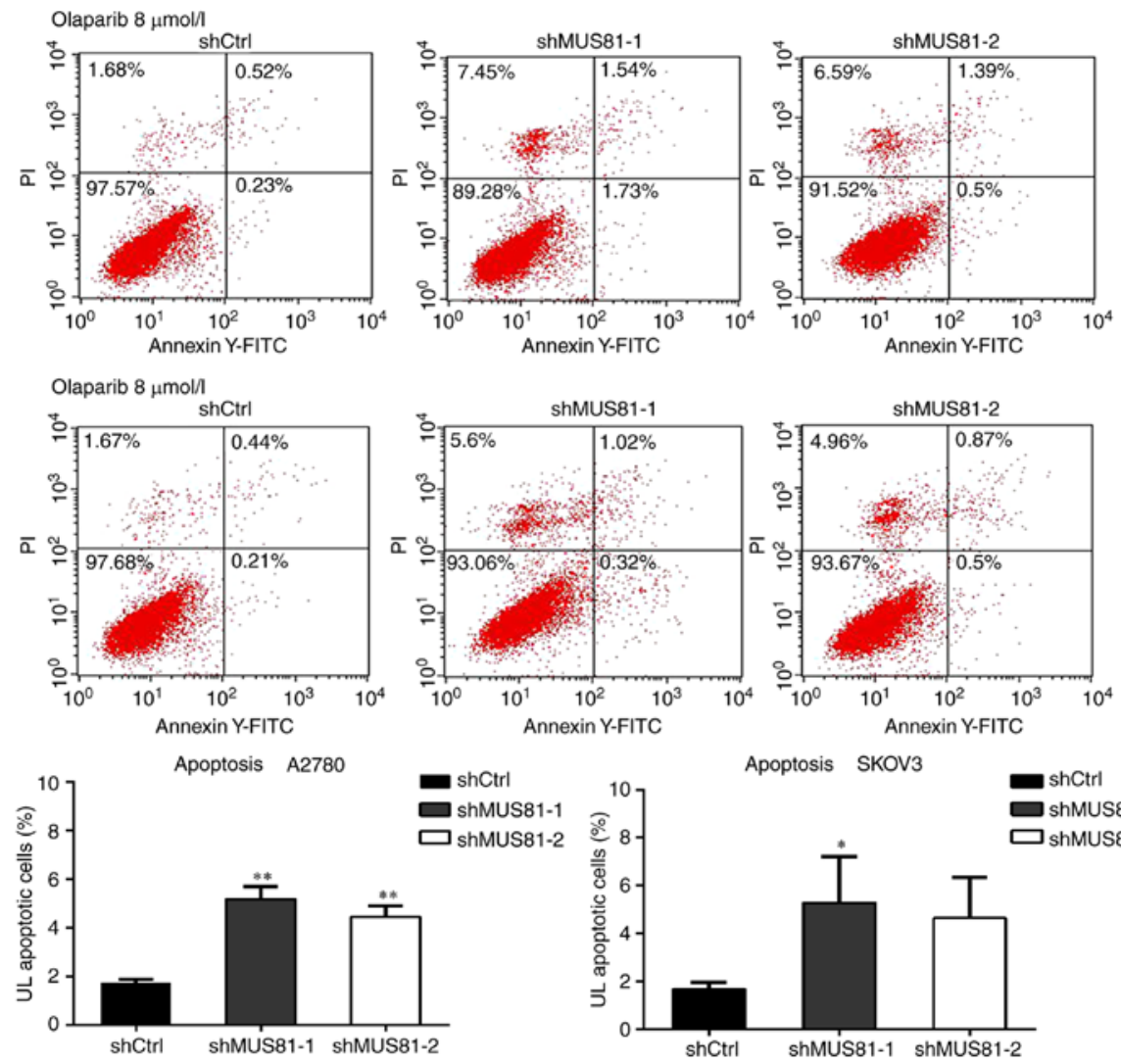

Figure 6. The effect of olaparib on cell cycle apoptosis. shCtrl and shMUS81 cells were exposed to $8 \mu \mathrm{mol} / 1$ olaparib for $48 \mathrm{~h}$. A2780 and SKOV3 cell apoptosis were analyzed by flow cytometry, and the data are presented as the mean \pm SD of three independent experiments.

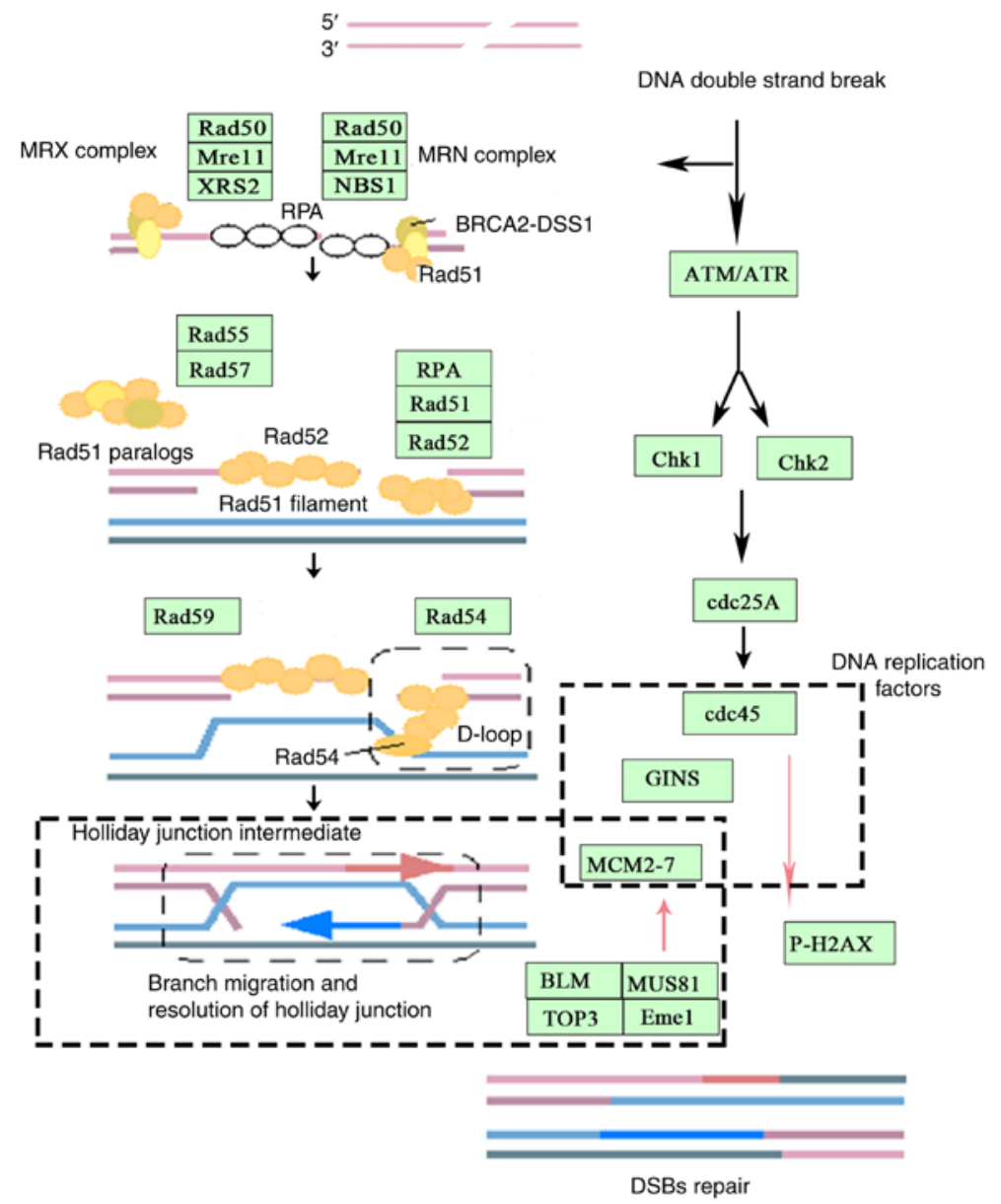

Figure 7. DNA replication and homologous recombination synergistically maintain genomic stability. Reference KEGG pathway. 


\section{Discussion}

Eukaryotic cells rely on accurate DNA replication and recombination to maintain genomic stability. HR and DNA replication mechanisms are required to coordinate activity at DNA DSBs and stalled replication forks (Fig. 7). Genomic instability resulting from either the loss or rearrangement of genetic material is closely related to the development of various human diseases, such as colorectal cancer and ovarian cancer $(14,15)$. DNA DSBs, which primarily result from the collapse of replication forks $(16,17)$, are often repaired through HR. The activity of HR machinery is closely related to the degree of injury repair. HR may lead to the formation of a DNA structure called Holliday junctions (HJs), which must be resolved for chromosome segregation. The first nuclear enzyme identified in both fission yeast and in human cells to possess a resolvase activity was MUS81, which exists as the MUS81-Eme1 complex. MUS81 is the catalytic subunit of a structural-specific endonuclease that has a preference for cleaving branched DNA substrates, including structures that are replication and recombination intermediates such as 3'flaps, 'Y'-shaped structures, D-loops and ' $\mathrm{X}$ '-shaped structures (HJs and HJ incisions) $(18,19)$. MUS81 also regulates the rate of DNA replication during normal cell growth by facilitating replication fork progression and reducing the frequency of replication initiation events. In the absence of MUS81 endonuclease activity, the rate of DNA synthesis declines, and the frequency of replication initiation events increases (20). The key step of the selection of the initiation replication site is the binding of the MCM helicase complex, which triggers replication (21). MCM2, as the regulatory protein of the replication initiation site, is an important component of MCM2-7 and plays an important role in the formation and decomposition of the replication fork $(22,23)$. Higher-order eukaryotes depend on high fidelity DNA replication and recombination mechanisms to maintain genomic stability. The dynamics of replisome components during fork collapse and restart are poorly understood. The repair of severe lesions in DNA, such as DSBs or stalled replication forks, requires coordinated activities of HR and DNA replication machineries (24). Growing evidence has also indicated that so-called 'accessory proteins' in two systems, such as helper protein RAD52, BRCA, and DNA replication proteins MCM2-7 and RAD54, are necessary for the effective coupling of recombination to replication that is essential for restoring genomic integrity after severe DNA damage (25). We discovered that both MUS81 and MCM2 act on the replication fork during HR, which in turn, activates MCM2 helicase when MUS81 endonuclease activity is blocked, leading to an unstable replication fork that dissociates from the DNA strand and inhibits the reformation of the DNA double strand.

HR is an important pathway contributing to DNA repair and HR defects are found in many cancers, such as ovarian cancer and hereditary breast cancer (26). Tumor cells with HR repair defects are sensitive to chemotherapeutic agents (cisplatin, carboplatin, etc.) $(27,28)$. Recent studies have indicated that HR-defective tumors are also exquisitely sensitive to novel agents, such as PARP inhibitors (29,30). PARP inhibitors have been identified as a new class of drugs for the treatment of ovarian cancer, and they induce significant effects in ovarian tumors with BRCA mutations. Olaparib is a recently discovered targeting drug for the treatment of ovarian cancer and exerts favorable effects against EOC with BRCA mutations. PARP inhibitors have good sensitivity for HR-deficient BRCA mutants in ovarian cancer and showed significant therapeutic effects in the treatment of recurrent EOC in phase I clinical trials (10). We found that the inhibitory effect of olaparib was enhanced in BRCA wild-type ovarian cancer cells and after downregulation of MUS81 DSBs. In BRCA wild-type ovarian cancer cells, DSBs were caused by DNA accumulation due to the inhibition of PARPs. The BRCA gene is involved in the HR pathway, but downregulation of MUS81 can inhibit the activity of endonucleases, reducing the efficiency of HR, and thus, they cannot complete DSB repair or inhibit tumor cell growth. Consequently, cell cycle and apoptosis assays revealed that olaparib affected cell cycle arrest in the $\mathrm{S}$ phase and increased cell apoptosis in MUS81 $1^{+}$BRCA-ovarian cancer cells.

We further found that downregulation of MUS81 improved the chemical sensitivity to olaparib. Treatment of cells with olaparib increases MCM2 expression, and activation of MCM2 is involved in regulation of the cell cycle and apoptosis. HR is required to use the homologous sequence of the intact sister chromatid as a template for its repair. Therefore, the repair process of HR is generally limited from the $S$ to the G2 phase of the cell cycle. MCM2, as a cyclin-dependent protein, is present in the G1 phase in the form of the MCM2-7 complex in a dormant state (31). During S phase, many factors are activated by binding to the MCM2-7 helicase or are chemically modified to activate the replication site (32). Helicase activation depends on the replisome component of Cdc45 (33), in addition to MCM2-7 and concomitant GINS assembly (together with the formation of CMG) (34). Activation of MCM2-7 with Cdc45 and GINS (CMG) is involved in the helicase, but inactivation of endonuclease activity results from the downregulation of MUS81, which is unable to form a 3 '-single-stranded end. The stalled replication fork is unable to form HJs and separate from the replication fork, causing the cell to arrest in the $\mathrm{S}$ phase. Furthermore, increasing MCM2 induces marked increases in Cdc45. Previous studies have shown that overexpression of $\mathrm{Cdc} 45$ in cells promotes cell apoptosis (35). Overexpression of Cdc45 significantly reduced the rate of replication fork extension by two-fold, and the replication fork was significantly asymmetric and arrested in the $\mathrm{S}$ phase. In addition, increased Cdc 45 leads to depletion of the single-stranded binding protein RPA and accumulation of single-stranded DNA. Single-stranded DNA accumulation is a marker of replication disaster, which results in decreases in the ATR/Chk1 pathway response, followed by activation of ATM/Chk2 via a replication fork break, which activates $\mathrm{pH} 2 \mathrm{AX}$ expression and promotes cell apoptosis (36).

We propose for the first time that MUS81 and MCM2 play important roles in the regulation of HR and clarify the dynamic effect between stalled replication forks and DNA replication during HR in DSB repair. Furthermore, the mechanisms of MCM2 activation in the regulation of DNA damage repair and DNA replication remain to be further explored.

In this study, the roles of MUS81 in the regulation of DNA damage repair in EOC and its potent effects on HR, the cell cycle and apoptosis via MCM2 activation were observed 
in vitro. These results suggest that MUS81 may be a new target of olaparib for the treatment of EOC, as it potently improved the sensitivity of BRCA wild-type EOC to PARP inhibitors, and that MUS81 can be used in new therapeutic approaches for future ovarian cancer treatment.

\section{Acknowledgements}

The present study was supported by a grant from the National Natural Science Foundation of China (grant no. NSF-81572552).

\section{Competing interests}

The authors declare that they have no competing interests.

\section{References}

1. Salani R and Bristow RE: Surgical management of epithelial ovarian cancer. Clin Obstet Gynecol 55: 75-95, 2012.

2. Haber JE and Heyer WD: The fuss about Mus81. Cell 107: 551-554, 2001

3. Boddy MN,Lopez-Girona A, Shanahan P, Interthal H, Heyer WD and Russell P: Damage tolerance protein Mus81 associates with the FHA1 domain of checkpoint kinase Cds1. Mol Cell Bio 20: 8758-8766, 2000.

4. Hanada K, Budzowska M, Davies SL, van Drunen E, Onizawa H, Beverloo HB, Maas A, Essers J, Hickson ID and Kanaar R: The structure-specific endonuclease Mus81 contributes to replication restart by generating double-strand DNA breaks. Nat Struct Mol Biol 14: 1096-1104, 2007.

5. Sarbajna S and West SC: Holliday junction processing enzymes as guardians of genome stability. Trends Biochem Sci 39: 409-419, 2014.

6. Xie S, Zheng H, Wen X, Sun J, Wang Y, Gao X, Guo L and Lu R: MUS81 is associated with cell proliferation and cisplatin sensitivity in serous ovarian cancer. Biochem Bioph Res Commun 476: 493-500, 2016

7. McPherson JP, Lemmers B, Chahwan R, Pamidi A, Migon E, Matysiak-Zablocki E, Moynahan ME, Essers J, Hanada K, Poonepalli A, et al: Involvement of mammalian Mus81 in genome integrity and tumor suppression. Science 304: 1822-1826, 2004.

8. Dendouga N, Gao H, Moechars D, Janicot M, Vialard J and McGowan CH: Disruption of murine Mus81 increases genomic instability and DNA damage sensitivity but does not promote tumorigenesis. Mol Cell Biol 25: 7569-7579, 2005.

9. Ledermann JA: PARP inhibitors in ovarian cancer. Ann Oncol 27 (Suppl 1): i40-i44, 2016.

10. Gadducci A and Guerrieri ME: PARP inhibitors in epithelial ovarian cancer: State of art and perspectives of clinical research. Anticancer Res 36: 2055-2064, 2016.

11. Liu FW and Tewari KS: New targeted agents in gynecologic cancers: Synthetic lethality, homologous recombination deficiency, and PARP inhibitors. Curr Treat Option Oncol 17: 12, 2016.

12. McLachlan $\mathrm{J}$ and Banerjee S: Olaparib for the treatment of epithelial ovarian cancer. Expert Opin Pharmacother 17: 995-1003, 2016.

13. Gunderson CC and Moore KN: Olaparib: An oral PARP-1 and PARP-2 inhibitor with promising activity in ovarian cancer. Future Oncol 11: 747-757, 2015.

14. Negrini S, Gorgoulis VG and Halazonetis TD: Genomic instability - an evolving hallmark of cancer. Nat Rev Mol Cell Biol 11: 220-228, 2010.

15. Grady WM: Genomic instability and colon cancer. Cancer Metastasis Rev 23: 11-27, 2004.
16. Tercero JA, Longhese MP and Diffley JF: A central role for DNA replication forks in checkpoint activation and response. Mol Cell 11: 1323-1336, 2003.

17. Sabatinos SA, Green MD and Forsburg SL: Continued DNA synthesis in replication checkpoint mutants leads to fork collapse. Mol Cell Biol 32: 4986-4997, 2012.

18. Chen XB, Melchionna R, Denis CM, Gaillard PH, Blasina A, Van de Weyer I, Boddy MN, Russell P, Vialard J and McGowan CH: Human Mus81-associated endonuclease cleaves holliday junctions in vitro. Mol Cell 8: 1117-1127, 2001.

19. Boddy MN, Gaillard PH, McDonald WH, Shanahan P, Yates JR III and Russell P: Mus81-Eme1 are essential components of a holliday junction resolvase. Cell 107: 537-548, 2001.

20. Fu H, Martin MM, Regairaz M, Huang L, You Y, Lin CM, Ryan M, Kim R, Shimura T, Pommier Y and Aladjem MI: The DNA repair endonuclease Mus81 facilitates fast DNA replication in the absence of exogenous damage. Nat Commun 6: 6746, 2015.

21. Blow JJ and Dutta A: Preventing re-replication of chromosomal DNA. Nat Rev Mol Cell Biol 6: 476-486, 2005.

22. Sun J, Fernandez-Cid A, Riera A, Tognetti S, Yuan Z, Stillman B, Speck C and Li H: Structural and mechanistic insights into Mcm2-7 double-hexamer assembly and function. Genes Dev 28: 2291-2303, 2014

23. Cortez D, Glick G and Elledge SJ: Minichromosome maintenance proteins are direct targets of the ATM and ATR checkpoint kinases. Proc Natl Acad Sci USA 101: 10078-10083, 2004.

24. Liu M, Ba Z, Costa-Nunes P, Wei W, Li L, Kong F, Li Y, Chai J, Pontes $\mathrm{O}$ and Qi Y: IDN2 interacts with RPA and facilitates DNA double-strand break repair by homologous recombination in arabidopsis. Plant Cell 29: 589-599, 2017.

25. Maher RL, Branagan AM and Morrical SW: Coordination of DNA replication and recombination activities in the maintenance of genome stability. J Cell Biochem 112: 2672-2682, 2011.

26. Mao P, Liu J, Zhang Z, Zhang H, Liu H, Gao S, Rong YS and Zhao Y: Homologous recombination-dependent repair of telomeric DSBs in proliferating human cells. Nat Commun 7: 12154, 2016.

27. Tan DS, Rothermundt C, Thomas K, Bancroft E, Eeles R, Shanley S, Ardern-Jones A, Norman A, Kaye SB and Gore ME: 'BRCAness' syndrome in ovarian cancer: A case-control study describing the clinical features and outcome of patients with epithelial ovarian cancer associated with BRCA1 and BRCA2 mutations. J Clin Oncol 26: 5530-5536, 2008.

28. Krajewska M, Fehrmann RS, de Vries EG and van Vugt MA: Regulators of homologous recombination repair as novel targets for cancer treatment. Front Genet 6: 96, 2015.

29. Bryant HE, Schultz N, Thomas HD, Parker KM, Flower D, Lopez E, Kyle S, Meuth M, Curtin NJ and Helleday T: Specific killing of BRCA2-deficient tumours with inhibitors of poly(ADPribose) polymerase. Nature 434: 913-917, 2005.

30. Farmer H, McCabe N, Lord CJ, Tutt AN, Johnson DA, Richardson TB, Santarosa M, Dillon KJ, Hickson I, Knights C, et al: Targeting the DNA repair defect in BRCA mutant cells as a therapeutic strategy. Nature 434: 917-921, 2005.

31. Remus D, Beuron F, Tolun G, Griffith JD, Morris EP and Diffley JF: Concerted loading of MCM2-7 double hexamers around DNA during DNA replication origin licensing. Cell 139: 719-730, 2009.

32. Labib K: How do Cdc7 and cyclin-dependent kinases trigger the initiation of chromosome replication in eukaryotic cells? Genes Dev 24: 1208-1219, 2010.

33. Tercero JA, Labib K and Diffley JF: DNA synthesis at individual replication forks requires the essential initiation factor Cdc45p. EMBO J 19: 2082-2093, 2000.

34. Moyer SE, Lewis PW and Botchan MR: Isolation of the Cdc45/Mcm2-7/GINS (CMG) complex, a candidate for the eukaryotic DNA replication fork helicase. Proc Natl Acad Sci USA 103: 10236-10241, 2006.

35. Rodriguez R, Gagou ME and Meuth M: Apoptosis induced by replication inhibitors in Chk1-depleted cells is dependent upon the helicase cofactor Cdc45. Cell Death Differ 15: 889-898, 2008.

36. Köhler C, Koalick D, Fabricius A, Parplys AC, Borgmann K, Pospiech $\mathrm{H}$ and Grosse $\mathrm{F}$ : Cdc45 is limiting for replication initiation in humans. Cell Cycle 15: 974-985, 2016. 